\title{
Multiple Classifier Systems in Texton-Based Approach for the Classification of CT Images of Lung
}

\author{
Mehrdad J. Gangeh ${ }^{1}$, Lauge Sørensen ${ }^{2}$, Saher B. Shaker ${ }^{3}$, \\ Mohamed S. Kamel ${ }^{1}$, and Marleen de Bruijne ${ }^{2,4}$ \\ ${ }^{1}$ Department of Electrical and Computer Engineering, University of Waterloo, Canada, \\ \{mgangeh, mkamel\}@ pami.uwaterloo.ca \\ ${ }^{2}$ Department of Computer Science, University of Copenhagen, Denmark, \\ \{lauges, marleen\}@diku.dk \\ ${ }^{3}$ Department of Respiratory Medicine, Gentofte University Hospital, Hellerup, Denmark \\ ${ }^{4}$ Biomedical Imaging Group Rotterdam, Erasmus MC, the Netherlands
}

\begin{abstract}
In this paper, we propose using texton signatures based on raw pixel representation along with a parallel multiple classifier system for the classification of emphysema in computed tomography images of the lung. The multiple classifier system is composed of support vector machines on the texton signatures as base classifiers and combines their decisions using product rule. The proposed approach is tested on 168 annotated regions of interest consisting of normal tissue, centrilobular emphysema, and paraseptal emphysema. Textonbased approach in texture classification mainly has two parameters, i.e., texton size and $k$ value in $k$-means. Our results show that while aggregation of single decisions by SVMs over various $k$ values using multiple classifier systems helps to improve the results compared to single SVMs, combining over different texton sizes is not beneficial. The performance of the proposed system, with an accuracy of $95 \%$, is similar to a recently proposed approach based on local binary patterns, which performs almost the best among other approaches in the literature.
\end{abstract}

\section{Introduction}

Texture-based pixel classification in computed tomography (CT) images of the lung is an emerging and promising tool for quantitative analysis of lung diseases such as emphysema, one of the main components of chronic obstructive lung disease (COPD). Emphysema, which is characterized by the loss of lung tissue, is visible in CT images as textural patterns comprising low intensity blobs or low attenuation areas of varying sizes (refer to Fig. 1 for some examples). Supervised classification using texture features allows for taking the emphysema texture into account. This was introduced in [1], and since then, various features have been used for the classification of emphysema and other abnormalities in lung CT images, including moments of filter response histograms from filter banks based on Gaussian derivatives [2], measures on gray-level co-occurrence matrices (GLCM), measures on gray-level runlength matrices (GLRLM), and moments of the attenuation histogram [1, 3, 4]. 
It has been shown, recently, that small-sized local operators like local binary patterns (LBP) [5] and patch representation of small local neighborhood in textonbased approaches [6] yield excellent texture classification performance on standard texture databases. Small-sized local operators are especially desirable in situations where the region of interest (ROI) is rather small, which is often the case in texture analysis in medical imaging, where pathology can be localized in small areas. This is because of two reasons: first, convolution with large support filter banks suffers from boundary effects; second, more patches can be extracted using small-sized local operators that makes the estimation of image statistics more reliable [6]. It is also shown in [7] that using support vector machines (SVMs) instead of $k$ nearest neighbor $(k$-NN) classifier, which is used in $[6,8]$ can improve the performance of texton-based approaches even further.

In our previous work [9], we proposed to use small patch representation in textonbased approaches for the classification of emphysema in CT images of the lung. This approach mainly consists of two learning steps: first an unsupervised step using $k$ means to construct a codebook of textons. Second, a supervised step in which the model is learned by obtaining a histogram of textons to represent each region of interest (ROI). There are two main parameters in these two steps, $k$ in $k$-means and texton size (TS), i.e., the size of patches extracted from the ROIs.

In general, the optimal parameters can vary regionally within the lung and from patient to patient, depending on the intrinsic scale and complexity of the texture patterns. Hence, it is not known a priori which texton size or $k$ value in $k$-means yields the best performance [10]. Hence, one possibility is to represent the ROIs using various $k$ and TS parameters that yield several feature subsets. There are several approaches in the literature to incorporate the information from all these feature subsets into a single decision system. These are including combined feature space (CFS), in which the feature subsets are fused to construct a single feature space and then submit it to a single classifier [11]; multiple classifier systems (MCS), where each feature subset is submitted to a so called base classifier (BC) and the decision by these $\mathrm{BCs}$ are combined into a single decision [11]; and multiple kernel learning (MKL), a system of multiple support vector machines (SVMs), each of which with its own kernel $[12,13]$. In MKL, the weights used for combining the decisions of the SVMs are optimized within the SVM optimization which leads to a quadratic optimization problem with quadratic constraint.

In this paper we propose to use multiple classifier systems with SVM as base classifier to aggregate the decisions made by the base classifiers using features (here histograms) at multiple texton sizes or multiple $k$ values. However, all SVMs use the same kernel, a radial basis function (RBF) kernel, and the decisions by these SVMs are combined using a fixed rule such as product rule. Our results show that the performance of the classification system using multiple classifier systems produces better results than single base classifiers and provides a means for making use of the information at various parameters of the approach. It also yields similar to or better results than the current approaches in the literature for the same application such as local binary patterns (LBPs) or filter bank approaches. 


\section{Texton-Based Approach}

In this section, we first briefly explain texton-based approach in texture classification. Then we present multiple classifier systems at multiple texton-based features as a means to aggregate feature subsets obtained at various texton sizes or $k$ values.

\subsection{Texton-Based Texture Classification}

The basic idea of textons was first introduced by Julesz as the elements of texture perception [14]. However, it took sometime before this idea could be developed into a texture classification system as proposed in [15]. This technique was further improved by Cula and Dana [16] and also Varma and Zisserman [6, 8] that yielded higher performance on standard texture databases.

There are three main representations associated with the texton-based approach in the literature, i.e., filter banks [8, 15, 16], raw pixel representation [6], and Markov random field (MRF) representation, where the central pixel in a neighborhood is modeled using the neighboring pixels [6]. However, irrespective of the representation used to describe local image information, the texton-based approach consists of learning and classification stages [6]. The learning stage, in turn, is divided into three steps: 1) construction of a codebook of textons using an unsupervised (clustering) algorithm such as $k$-means; 2) learning texton histograms from the training set; and 3 ) training a classifier such as SVM using texton histograms obtained in step two. In the classification stage, the class of a test image is determined by submission of the histogram of textons in the test image to the classifier trained in the learning stage.

To construct the texton codebook, small-sized local patches are randomly extracted from each image ${ }^{1}$ in the training set. These small patches are then converted to the appropriate representation such as filter banks or raw pixels. Eventually, they are aggregated over all images in a class and clustered using a clustering algorithm such as $k$-means. The cluster centers obtained form a dictionary of textons to represent the class of textures. It will be used as the codebook of textons in the next step. The size of the dictionary depends on the number of cluster centers, e.g., $k$ in $k$-means algorithm as well as the number of classes. For example, for a three-class problem with $k$ of $30,3 \times 30=90$ textons are generated in the codebook. Fig. 1 displays sample images of lung CT ROIs used in this paper as well as a codebook of 90 textons computed over all ROIs using the texton size of $9 \times 9$ pixels and $k=30$.

The second step in the learning stage is supervised, in which a histogram of textons is found for each image in the training set as a model (feature set) to represent this image. To find this histogram, small patches of the same size as in the unsupervised step are extracted by sliding a window over each training image in a class. These patches are then converted to the appropriate representation as used in the previous step. Finally, a histogram of textons is computed for the image by comparing each and every patch representation in that image with all textons in the dictionary using a similarity measure to find the closest match and updating the corresponding histogram bin based on the closest match found. The histograms are normalized and used as the

\footnotetext{
${ }^{1}$ In this paper image and region of interest (ROI) of the lung are used interchangeably.
} 
feature sets for the images in the training set and employed for training a classifier such as a support vector machine (SVM) as the third step of the learning. Left and middle diagrams in Fig. 2 illustrate the construction of the codebook and learning the model in a texton-based classification system using raw pixel representation.

In the classification stage, to classify a test image, the same steps as in the learning stage are followed to find the features for the test image. This includes extraction of small patches from each test image in a class, converting the patches to the appropriate representation, finding the closest match to these patches from the dictionary, and computing the normalized histogram of obtained closest textons to define a feature vector for the image. The trained classifier in the learning stage is subsequently used to find the class of the test image. In SVM, a RBF kernel as given in (1) is used as it is recommended as the first kernel choice in [17]. In (1), $\gamma$ is the kernel width and $\mathbf{x}_{i}$ and $\mathbf{x}_{\mathrm{j}}$ are two sample patterns.

$$
K\left(\mathbf{x}_{i}, \mathbf{x}_{j}\right)=e^{-\gamma\left\|\mathbf{x}_{i}-\mathbf{x}_{j}\right\|^{2}}
$$

\subsection{Multiple Classifier Systems}

The learning stage in texton-based approach generates an $n$-dimensional vector $\mathbf{h}^{(i)}=\left[h_{1}, \ldots, h_{n}\right] \in \mathbb{R}^{n}, i=1, \ldots, m$ for each ROI, where $n$ is the number of bins in the histogram of textons and $m$ is the total number of texton-sizes or $k$ values for which the model is learned. Each $\mathbf{h}^{(i)}$ is considered as a feature subset obtained at a specific texton size or $k$ value and they can be composed into a single feature space $\mathbf{h}=\left[\mathbf{h}^{(1)}, \ldots, \mathbf{h}^{(m)}\right]$, which is called distinct pattern representation (DPR) [18].

We propose here to submit this DPR to an ensemble of classifiers [11]:

$$
\Gamma=\left\{D_{1}, \ldots, D_{m}\right\}, \quad \Gamma: \mathbb{R}^{n \times m} \rightarrow \Omega^{m}
$$

where, $\Gamma$ is the ensemble with $D_{i}: \mathbb{R}^{n} \rightarrow \Omega, i=1, \ldots, m$, as base classifier (BC) trained on each feature subset $\mathbf{h}^{(\mathrm{i})} \in \mathbb{R}^{n}, i=1, \ldots, m$ and $\Omega=\left\{\omega_{1}, \ldots, \omega_{c}\right\}$ is the set of class labels.
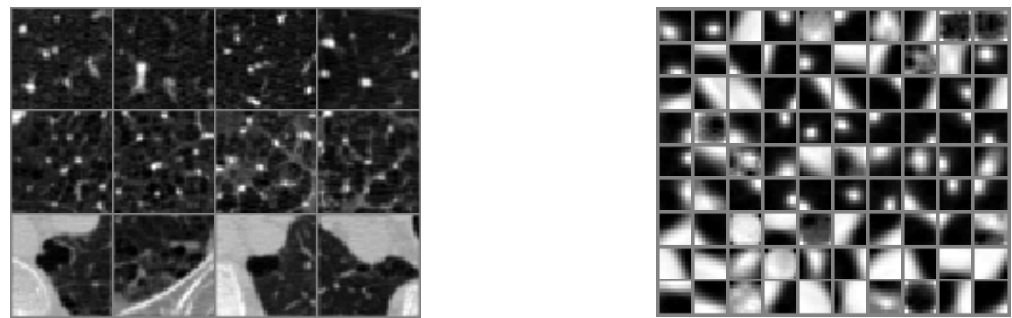

Fig. 1. Sample ROIs of size $50 \times 50$ pixels (left) in three classes, i.e., normal lung (top left row), CLE (middle left row), and PSE (bottom left row). The constructed codebook using texton sizes of $9 \times 9$ pixels and $k=30$ in $k$-means (right). 


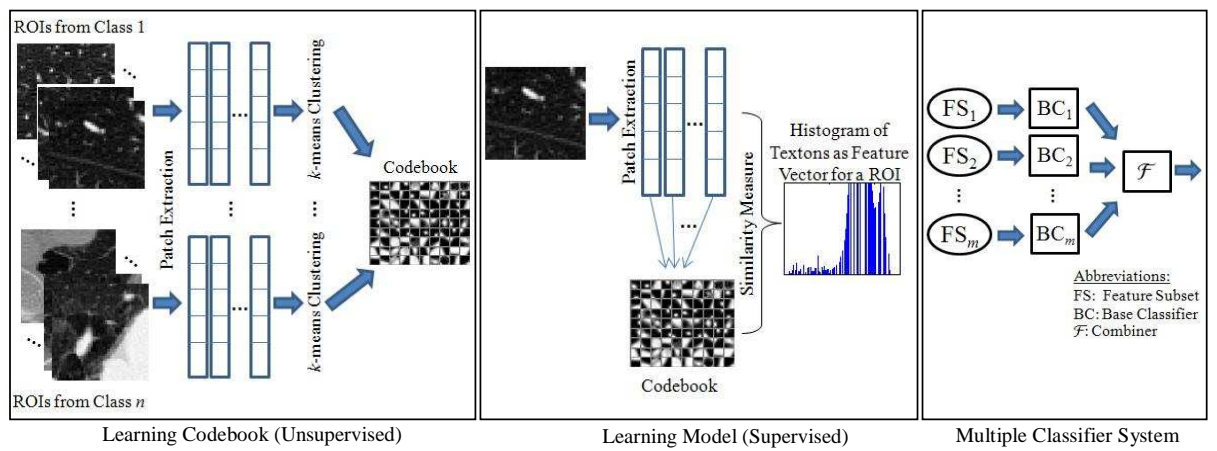

Fig. 2. The illustration of different stages of the proposed system using multiple classifier systems and texton signatures: the generation of texton codebooks using $k$-means clustering (left), the generation of features by computing the texton histograms (middle), and parallel multiple classifier system to aggregate the decisions by single base classifiers (right).

The decisions made by these BCs are subsequently fused by the aggregation function $\mathcal{F}$ to yield a single decision on the class of the pattern submitted for classification such that $\mathcal{F}: \Omega^{m} \rightarrow \Omega$.

There are three main structures of multiple classifier systems (MCS), i.e., stacked MCS with the same feature space for all BCs; parallel MCS with a distinct feature space for each BC; and sequential MCS in which the output of each BC is given to the next one. Here since the feature subset $\mathbf{h}^{(i)}$ given to each BC is different, parallel MCS is a natural choice. The right diagram in Fig. 2 illustrates the structure of the proposed multiple classifier system.

\section{Experimental Setup}

Data Preparation. Emphysema is often classified into various subtypes based on morphology [19]. In this work, we focus on the two subtypes related to smoking, namely, centrilobular emphysema (CLE), defined as multiple small low-attenuation areas and paraseptal emphysema (PSE), defined as multiple low-attenuation areas in a single layer along the pleura often surrounded by interlobular septa that is visible as thin white walls. The data used for the experiments is the same as in $[9,20,21]$ and comprises 168 ROIs, of size $50 \times 50$ pixels, representing the following three classes: normal tissue (NT) (59 ROIs), CLE (50 ROIs), and PSE (59 ROIs). The ROIs are extracted from 75 thin-slice pulmonary CT images of 25 different subjects where the leading pattern was obtained as the consensus visual assessment by two experienced readers. The NT ROIs are from healthy non-smokers while the emphysema ROIs are from smokers. CT was performed using GE equipment (LightSpeed QX/i; GE Medical Systems, Milwaukee, WI, USA) with four detector rows, using the following parameters: in-plane resolution $0.78 \times 0.78 \mathrm{~mm}, 1.25 \mathrm{~mm}$ slice thickness, tube voltage $140 \mathrm{kV}$, and tube current $200 \mathrm{mAs}$. The slices were reconstructed using a high spatial resolution (bone) algorithm. 
Computation of Texton-Based Features. The codebook of textons is constructed by extracting 500 random patches from each ROI in the training set. Patch sizes of $3 \times 3$ to $9 \times 9$ pixels are used in the experiments. Raw pixel representation is used. Since in CT images, the mean of the intensity in the images indicate a physical property of the tissue, the mean of the ROIs are not removed. The patches extracted from different ROIs of each class are given to $k$-means clustering algorithm to find the codebook. Five different values of $k$, i.e., $k=10$ to $k=50$ are tested in the experiments. After construction of texton codebook, texton frequency histograms of texture images are computed to find the model. In this stage, small overlapping patches with the same size as what was used in the clustering stage are extracted from top left to bottom right of each ROI. As in the clustering stage, no filter bank is used and raw pixel representation is considered. The Euclidean distance between the resulting textons (collection of small patches) and textons in the codebook is computed in order to identify the most similar texton in the codebook and the corresponding histogram of textons is updated. Normalized histograms are used as the feature subsets $\mathbf{h}^{(i)}$.

Classifier and Evaluation. The evaluation of the classification system is performed using leave-one-subject-out. This means that all the ROIs of one subject (patient) are used as the test set and the remaining ROIs as the training set. A parallel multiple classifier system with SVM as base classifier (BC) is used. It is shown in [9] that SVM performs better than $k$-NN in the classification of emphysema and hence SVM is used as the BC in our experiments. Product combiner is selected as the aggregation function $\mathcal{F}$ as our preliminary experiments show that it performs almost the best among other combiners including majority voting, mean, and max combiners. The crucial issue in using SVM is finding a suitable kernel and the optimum trade-off parameter $C$. RBF kernel is selected for the SVMs and its optimum kernel width, i.e., $\gamma$ in (1) as well as the trade-off parameter $C$ are found by a grid search on the training set at each specific texton size and $k$ value. To avoid too much computational cost for this grid search, 5-fold cross-validation at patient level (instead of leave-one-subjectout) is performed on the training set. This means that the training set is divided into five folds at patient level. One fold is used as the validation set and the remaining as the training set. Since the codebook has to be only constructed on the training set, we need to construct the codebook each time on the four folds used at this cross validation. We have, thus, repeated the experiments 10 times and averaged the results as there is a variation in the patches extracted each time for the construction of codebook.

\section{Results}

In this section, we first present the results for texton-based texture classification system using one single SVM as classifier with the parameters chosen as explained in previous section. Then the results of aggregation over different values of $k$ with fixed texton-size and also aggregation over different texton sizes with fixed $k$ are presented. Eventually, the comparison between single SVM and multiple classifier system is presented followed by the comparison with other techniques reported in the literature. 
The results for using one single SVM are shown in Table 1 for various texton sizes and $k$ values in $k$-means. The last row and last column on this table show the results of using multiple classifier systems with product combiner that aggregates the decisions of single SVMs at various texton sizes or $k$ values, respectively. These results are also shown graphically in Fig. 3 to make the comparison between single SVM and multiple classifier systems easier. As can be seen from top graph in Fig. 3, aggregation over various $k$ values almost always yields better results than single $k$ at the corresponding texton size. However, the bottom graph in Fig. 3 reveals that combining over various texton-sizes at the same $k$ value does not produce better results than the best single SVM.

Comparison with Other Techniques. The comparison is made between the proposed texton-based classification system using multiple classifier systems with aggregation over $k$ values and the results published in [21]. Since the same data as in [21] is used in our experiments, the results are directly comparable. In [21], the results are provided for several approaches among which we consider a filter bank approach using moments of histograms and an approach based on the LBP operators as follows:

1) GFB1 (Gaussian filter bank 1): using the moments of histogram computed on the outputs of convolved Gaussian filter banks with four rotation invariant filters obtained from linear combination of Gaussian derivatives at five scales.

2) LBP2: joint 2D LBP and intensity histograms.

The reader may refer to [21] for more information on these two approaches and also for further comparison with other techniques described therein. Moments of histograms computed on the outputs of Gaussian derivatives are one of the most common approaches in the literature for the classification of CT images of lung [2]. On the other hand, LBP2 reaches the best results among others in [21]. The results based on the above techniques are provided in Table 2 along with the best result obtained from the proposed approach based on texton signatures and multiple classifier system with aggregation over different $k$ values using product combiner.

The confusion matrices for LBP2 and our best results are provided in Table 2. The proposed approach attains performance similar to LBP2 and McNemar's test also does not indicate significant difference $(p=0.75)$. The specificity of texton-based using MCS and LBP2 approaches are $96.61 \%$ and $93.33 \%$, while their sensitivity are 95.37\% and 97.25\%, respectively (when comparing NT versus CLE and PSE).

Table 1. The results of texton-based classification system on CT images of lung used in this paper for $k$ values of 10 to 50 and texton sizes (TS) of $3 \times 3$ to $9 \times 9$ pixels using a single SVM.

\begin{tabular}{|c|c|c|c|c|c|c|}
\hline Texton Size & $k=10$ & $k=20$ & $k=\mathbf{3 0}$ & $k=40$ & $k=\mathbf{5 0}$ & $\begin{array}{c}\text { Aggregation } \\
\text { over } k\end{array}$ \\
\hline $3 \times 3$ & $93.5 \pm 1.1$ & $93.2 \pm 1.2$ & $94.7 \pm 1.4$ & $93.0 \pm 1.0$ & $91.7 \pm 1.1$ & $94.5 \pm 0.6$ \\
\hline $4 \times 4$ & $92.9 \pm 0.7$ & $93.6 \pm 1.2$ & $93.5 \pm 1.3$ & $94.1 \pm 1.3$ & $94.2 \pm 0.9$ & $95.0 \pm 0.6$ \\
\hline $5 \times 5$ & $92.4 \pm 1.0$ & $91.7 \pm 0.9$ & $92.6 \pm 0.9$ & $92.7 \pm 1.2$ & $93.8 \pm 1.3$ & $94.2 \pm 0.4$ \\
\hline $6 \times 6$ & $91.7 \pm 1.3$ & $90.8 \pm 0.9$ & $91.8 \pm 1.5$ & $90.3 \pm 1.9$ & $90.5 \pm 1.4$ & $92.1 \pm 0.7$ \\
\hline $7 \times 7$ & $90.1 \pm 1.4$ & $91.1 \pm 1.3$ & $90.8 \pm 1.0$ & $89.8 \pm 1.6$ & $89.2 \pm 1.7$ & $91.1 \pm 0.7$ \\
\hline $8 \times 8$ & $88.8 \pm 1.7$ & $89.5 \pm 1.9$ & $91.1 \pm 0.9$ & $91.0 \pm 1.3$ & $89.6 \pm 1.8$ & $91.7 \pm 0.9$ \\
\hline $9 \times 9$ & $87.6 \pm 1.4$ & $88.8 \pm 1.5$ & $91.0 \pm 1.1$ & $89.8 \pm 1.7$ & $90.5 \pm 1.0$ & $90.8 \pm 0.9$ \\
\hline $\begin{array}{c}\text { Aggregation } \\
\text { over TS } \\
\end{array}$ & $92.1 \pm 0.6$ & $93.0 \pm 0.7$ & $93.5 \pm 1.0$ & $93.0 \pm 0.6$ & $92.8 \pm 0.8$ & \\
\hline
\end{tabular}


Table 2. The comparison between the best results obtained from the proposed approach and the results of other techniques on the same data (left); the confusion matrix of LBP2 (middle) and texton-based approach with multiple classifier systems (MCS) and SVM as base classifier (right).

\begin{tabular}{|c|c|c|c|c|c|c|c|c|c|}
\hline Technique & Accuracy & & \multicolumn{3}{|c|}{ Estimated Labels } & & \multicolumn{3}{|c|}{ Estimated Labels } \\
\hline$\overline{\text { GFB1 }}$ & 61.3 & True Labels & NT & CLE & PSE & True Labels & NT & CLE & PSE \\
\hline LBP2 & 95.2 & NT & 55 & 0 & 4 & NT & 57 & 0 & 2 \\
\hline & & CLE & 1 & 49 & 0 & CLE & 4 & 46 & 0 \\
\hline $\begin{array}{l}\text { Texton-based } \\
\text { using MCS }\end{array}$ & 95.0 & PSE & 2 & 1 & 56 & PSE & 2 & 0 & 57 \\
\hline
\end{tabular}
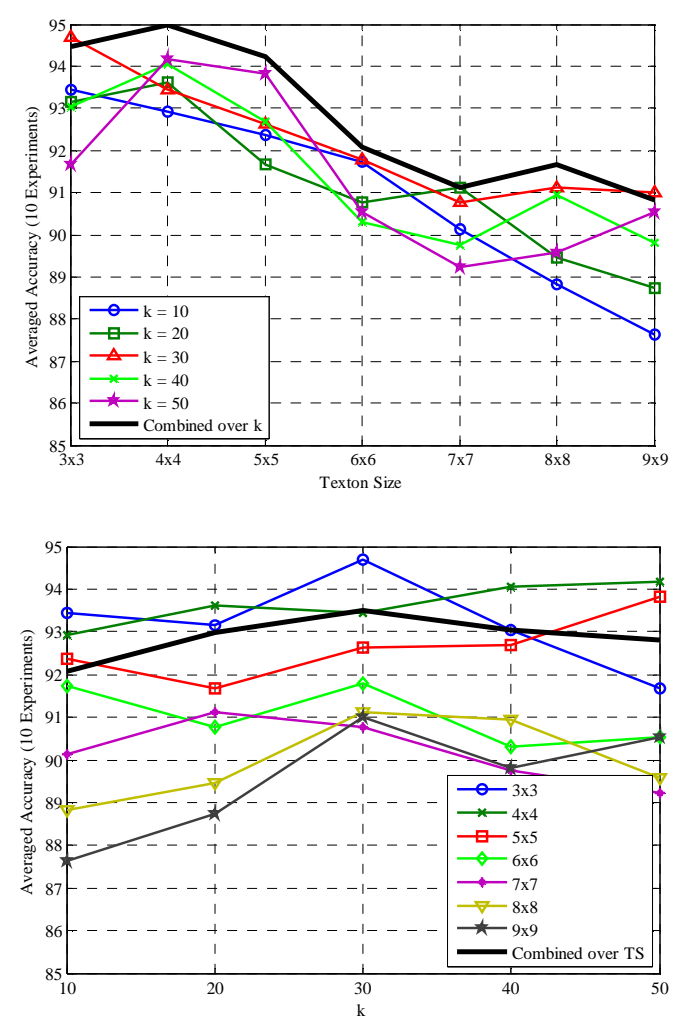

Fig. 3. The accuracy of the base classifiers and their combination on various texton sizes (TS) (top) and $k$ values (bottom).

\section{Discussions and Conclusion}

In this paper, multiple classifier systems along with texton signatures are proposed for the classification of CT images of lung. Our results on the dataset of 168 ROIs of 
CT images of lung shows that while texton-based approach using a single SVM has a satisfactory performance in this application; combining these single SVMs in a parallel structure over different $k$ values slightly improves the classification results.

From Table 1 and top graph in Fig. 3, it seems that increasing texton size degrades the performance of single SVMs. This could be because larger texton sizes lead to higher dimensional space for $k$-means, requiring more data for reliable clustering. At the same time, there will be fewer sub-patches available with larger texton size for learning the model as described in Section 2.1. The aggregation results on various texton sizes show no improvement. A close look at the outputs of single SVMs at the same $k$ value but different texton sizes reveals that most of the SVMs make the mistakes on the same ROIs and this means that there is a lack of diversity among the base classifiers. This explains why combining them do not improve the performance [11].

However, as can be seen from the bottom graph in Fig. 3 that displays the performance of single SVMs at a specific texton size at various $k$ values, no certain value of $k$ always yield the best results. At some texton sizes, larger $k$ produces the best results (for example at texton size $4 \times 4$ ) while at other texton sizes medium or small $k$ concludes best results. Since we do not know a priori which $k$ produces the best results as the optimal $k$ may vary from patient to patient depending on intrinsic scale and complexity of texture patterns, we aggregate over texton sizes at a specific $k$, which almost always produces better results than the best single SVM. By looking at the outputs of the base classifiers, it becomes clear that the diversity among them is higher than the previous case and this explains the improvement of the results by their combining.

Overall, we conclude that aggregating at smaller texton-sizes, for example $4 \times 4$ over different values of $k$ reasonably produces good results which are similar to or better than the results obtained from other approaches in the literature. Among these approaches is LBP2 [21], which mainly relies on LBP operators.

Using parallel multiple classifiers systems on texton signatures proposed here can also be extended to LBP approach. LBP operators also involve two parameters, i.e., the size of operator (scale), and the number of bins in the estimation of histogram. The decisions based on single operators can be aggregated over any of these two parameters to investigate possible improvements.

In comparing LBP and texton-based approaches provided in this paper, one should notice that LBP operators are, by design, invariant to monotonic intensity transformations. While this is desirable in some applications, in the classification of Lung CT images, the mean of intensity is important and this justifies poor performance of an approach based on merely LBP operators as it discards the mean of intensity in the ROIs [21]. Due to this drawback of LBPs, in [20, 21], the joint intensity and LBP histograms are considered (LBP2). This improves the performance of the LBPs in this application at the cost of adding to the complexity of the approach. Texton-based approach does not suffer from this problem as it is not invariant to intensity transformations. On the other hand, LBP operators can be considered as fixed textons which are chosen irrespective of the data. Texton-based approach, however, extracts the textons using the data. This adds to the complexity of textonbased approach as the unsupervised step in learning the dictionary of textons is an extra step in this approach comparing to the LBPs. The performance of texton-based 
approach and LBP2 is similar for the data used in this paper. Nevertheless, our conjecture is that the superiority of one approach to another is application dependent. If LBP operators can define a good representation for some data they conclude high performance while if texton-based approach can extract the textons accurately based on the training data, then it can yield high accuracy.

In future work, other classifiers that generate more diverse classification outputs than SVMs, such as decision trees, will be investigated. As mentioned above, in our experiments, SVMs as base classifiers lack diversity among themselves and, hence, combining their decisions does not yield significant improvement over best single SVM. We expect that decision trees, which are considered as ensemble of weak classifiers, generate more diverse outputs and their combination in a decision forest may conclude more improvement [22].

Also, in this paper, raw pixel representation is only used in texton-based approach. In some computer vision applications, it has been shown that building textons on the output of filter banks produces better accuracy [23, 24]. Although using raw pixel representation is computationally more attractive than using filter bank representation (as the intermediate step of convolving patches with the filter banks is not required), using filter banks in texton-based approach will be investigated in the future work for possible improvement of the results.

Acknowledgments. The funding from the Natural Sciences and Engineering Research Council (NSERC) of Canada under Canada Graduate Scholarship (CGS D3378361-2009) and Michael Smith Foreign Study Supplements (MSFSS) is gratefully acknowledged.

\section{References}

1. Uppaluri, R., Mitsa, T., Sonka, M., Hoffman, E.A., McLennan, G.: Quantification of Pulmonary Emphysema from Lung Computed Tomography Images. Amer. J. Respir. Crit. Care Med. 156(1) 248--254 (1997)

2. Sluimer, I.C., Prokop, M., Hartmann, I., van Ginneken, B.: Automated Classification of Hyperlucency, Fibrosis, Ground Glass, Solid, and Focal Lesions in High-Resolution CT of the Lung. Medical Physics 33(7) 2610--2620 (2006)

3. Chabat, F., Yang, G.Z., Hansell, D.M.: Obstructive Lung Diseases: Texture Classification for Differentiation at CT. Radiology 228(3) 871--877 (2003)

4. Xu, Y., Sonka, M., McLennan, G., Guo, J., Hoffman, E.A.: MDCT-based 3-D Texture Classification of Emphysema and Early Smoking Related Lung Pathologies. IEEE Trans. Med. Imag. 25(4) 464--475 (2006)

5. Ojala, T., Pietikainen, M., Maenpaa, T.: Multiresolution Gray-Scale and Rotation Invariant Texture Classification with Local Binary Patterns. IEEE Trans. Pattern Analysis and Machine Intelligence 24(7) 971--987 (2002)

6. Varma, M., Zisserman, A.: A Statistical Approach to Material Classification Using Image Patch Exemplars. IEEE Trans. Pattern Analysis and Machine Intelligence 31(11) 2032-2047 (2009)

7. Caputo, B., Hayman, E., Fritz, M., Eklundh, J.O.: Classifying Materials in the Real World. Image and Vision Computing 28(1) 150--163 (2010) 
8. Varma, M., Zisserman, A.: A Statistical Approach to Texture Classification from Single Images. International Journal of Computer Vision: Special Issue on Texture Analysis and Synthesis 62(1-2) 61--81 (2005)

9. Gangeh, M.J., Sørensen, L., Shaker, S.B., Kamel, M.S., de Bruijne, M., Loog, M.: A Texton-Based Approach for the Classification of Lung Parenchyma in CT Images. In: T. Jiang et al. (eds.) MICCAI 2010, LNCS, vol. 6363, pp. 596--603. Springer, Heidelberg (2010)

10. Garcia, M.A., Puig, D.: Supervised Texture Classification by Integration of Multiple Texture Methods and Evaluation Windows. Image and Vision Computing 25(7) 1091-1106 (2007)

11. Kuncheva, L.I.: Combining Pattern Classifiers Methods and Algorithms. John Wiley \& Sons, New Jersey (2004)

12. Lanckriet, G.R.G., Cristianini, N., Bartlett, P., Ghaoui, L.E., Jordan, M.I.: Learning the Kernel Matrix with Semidefinite Programming. Journal of Machine Learning Research 5(1) 27--72, 2005

13. Bach, F.R., Lanckriet, G.R.G., Jordan, M.I.: Multiple Kernel Learning, Conic Duality, and the SMO Algorithm. Proceedings of 21st International Conference of Machine Learning (ICML), 2004.

14. Julesz, B.: Textons, the Elements of Texture Perception, and Their Interactions. Nature 290(5802) 91--97 (1981)

15. Leung, T., Malik, J.: Representing and Recognizing the Visual Appearance of Materials Using Three-Dimensional Textons. International Journal of Computer Vision 43(1) 29--44 (2001)

16. Cula, O.G., Dana, K.J.: 3D Texture Recognition Using Bidirectional Feature Histograms International Journal of Computer Vision 59(1) 33--60 (2004)

17. Fan, R.E., Chen, P.H., Lin, C.J.: Working Set Selection Using the Second Order Information for Training SVM. Journal of Mach. Learning Research 6, 1889--1918 (2005)

18. Kittler, J., Hatef, M., Duin, R.P.W., Matas, J.: On Combining Classifiers. IEEE Trans. Pattern Analysis and Machine Intelligence 20(3), 226--239, 1998

19. Webb, W.R., Müller, N., Naidich, D.: High-Resolution CT of the Lung, Third Edition. Lippincott Williams \& Wilkins (2001)

20. Sørensen, L., Shaker, S.B., de Bruijne, M.: Texture Classification in Lung CT Using Local Binary Patterns. In: Metaxas, D., Axel, L., Fichtinger, G., Szekely, G. (eds.) MICCAI 2008, LNCS, vol. 5241, pp. 934--941. Springer, Heidelberg (2008)

21. Sørensen, L., Shaker, S.B., de Bruijne, M.: Quantitative Analysis of Pulmonary Emphysema Using Local Binary Patterns. IEEE Trans. Med. Imag. 29(2) 559--569 (2010)

22. Ho, T.K.: The Random Subspace Method for Constructing Decision Forests. IEEE Transactions on Pattern Analysis and Machine Intelligence 20(8) 832--844 (1998)

23. Tuzel, O., Yang, L., Meer, P., Foran, D.J.: Classification of Hematologic Malignancies Using Texton Signatures. Pattern Analysis and Applications 10(4) 277--290 (2007)

24. Zhong, C., Sun, Z., Tan, T.: Robust 3D Face Recognition Using Learned Visual Codebook. In: IEEE Conference on Computer Vision and Pattern Recognition (CVPR 2007), pp. 1--6 (2007) 